\title{
Three-Dimensional Evaluation of Similarity of Right and Left Knee Joints
}

\author{
Ki-Mo Jang, $\mathrm{MD}^{1}$, Jong-Hoon Park, $\mathrm{MD}^{1}$, Minho Chang, $\mathrm{PhD}^{2}$, Youngjun Kim, $\mathrm{PhD}^{3}$, Deukhee Lee, $\mathrm{PhD}^{3}$, \\ Sehyung Park, $\mathrm{PhD}^{3}$, and Joon Ho Wang, $\mathrm{MD}^{4}$ \\ ${ }^{1}$ Department of Orthopaedic Surgery, Anam Hospital, Korea University College of Medicine, Seoul; ${ }^{2}$ School of Mechanical Engineering, Korea University, Seoul; ${ }^{3}$ Center \\ for Bionics, Korea Institute of Science and Technology, Seoul; ${ }^{4}$ Department of Orthopaedic Surgery, Samsung Medical Center, Sungkyunkwan University School of \\ Medicine, Seoul, Koea
}

Purpose: The purpose of this study was to evaluate the anatomical similarity of three-dimensional (3D) morphometric parameters between right and left knees.

Materials and Methods: Ten fresh-frozen paired cadaveric knees were tested. Following dissection, footprint areas of the anterior cruciate ligament (ACL) and posterior cruciate ligament (PCL) were measured. Surface scanning was performed using a 3D scanner. Scanned data were reproduced and morphometric parameters were measured on specialized software. After making mirror models, we compared footprint center positions of the ACL and PCL of both sides and calculated the average deviation of 3D alignment between the right- and left-side models.

Results: No significant side-to-side differences were found in any morphometric parameters. Bony shapes displayed a side-to-side difference of $<1$ $\mathrm{mm}$. Distal femoral and proximal tibial volumes did not present side-to-side differences, either; the average 3D deviations of alignment between the right and left sides were $0.8 \pm 0.4 / 1.1 \pm 0.6 \mathrm{~mm}$ (distal femur/proximal tibia). Center-to-center distances between the right and left ACL footprints were $2.6 / 2.7 \mathrm{~mm}$ (femur/tibia) for the anteromedial bundle and $2.4 / 2.8 \mathrm{~mm}$ for the posterolateral bundle. They were $1.9 / 1.5 \mathrm{~mm}$ for the anterolateral bundle and 2.2/1.8 $\mathrm{mm}$ for the posteromedial bundle of the PCL.

Conclusions: There was a remarkable 3D morphometric similarity between right and left knees. Our results might support the concept of obtaining morphologic reference data from the uninvolved contralateral knee.

Keywords: Knee, Anterior cruciate ligament, Posterior cruciate ligament, Comparative anatomy, Similarity, Three-dimensional imaging

\section{Introduction}

Orthopedic surgeons commonly use anatomic information of the uninvolved side knee joint as a reference during preoperative planning and templating for knee joint arthroplasties ${ }^{1)}$. The

Received December 24, 2016; Revised (1st) March 5, 2017;

(2nd) March 21, 2017; Accepted March 23, 2017

Correspondence to: Joon Ho Wang, MD

Department of Orthopaedic Surgery, Samsung Medical Center,

Sungkyunkwan University School of Medicine, 81 Irwon-ro, Gangnamgu, Seoul 06351, Korea

Tel: +82-2-3410-3507, Fax: +82-2-3410-0061

E-mail: mdwang88@gmail.com

Source of funding: This study was supported by research fund from SMC-KIST Translational Research Program, 2014.

This is an Open Access article distributed under the terms of the Creative Commons Attribution Non-Commercial License (http://creativecommons.org/licenses/by-nc/4.0/) which permits unrestricted non-commercial use, distribution, and reproduction in any medium, provided the original work is properly cited. relative laxity of ligament-injured knee joints is determined by comparison with uninvolved contralateral knee joints. These practices are based on the assumption that both knee joints in the same subject are anatomically similar ${ }^{1-7)}$.

However, few studies have investigated the morphometric sideto-side differences in human knee joints. Furthermore, those studies reported inconsistent results for the clinical use of a sideto-side comparison ${ }^{1,2,8)}$. Dargel et al. ${ }^{1)}$ reported a good correlation of human knee joint anatomy between the right and the left sides. Although the study was a substantial report investigating various parameters, it performed a two-dimensional (2D) analysis, which might not be sufficiently sensitive to detect clinically important three-dimensional (3D) morphological knee joint characteristics. To our knowledge, there are only a few studies regarding 3D analyses of the morphometric side-to-side differences in human knee joints in the literature ${ }^{9-11)}$.

The use of computer assistance in knee joint surgery is em- 
phasized in arthroplasty, ligament reconstruction, and fracture surgery ${ }^{12-14)}$. Image-based computer-assisted surgeries could use non-involved contralateral anatomy as reference data if anatomical symmetry is confirmed ${ }^{1,2,15)}$. For example, if the footprint anatomy of each bundle of the anterior cruciate ligament (ACL) and posterior cruciate ligament (PCL) was symmetrical, the anatomical data of the uninjured knee joint could be applied as a reference for computer-assisted anatomical reconstruction. Thus, it is necessary to investigate the magnitude of side-to-side differences in the human knee joint for future surgeries as well as traditional practices in orthopedics.

The purpose of this study was to compare the 3D morphometric dimensions of distal femur and proximal tibia and footprint anatomy of the ACL and PCL between right and left knee joints using human cadavers. It was hypothesized that $3 \mathrm{D}$ knee joint morphology is symmetrical between right and left knees.

\section{Materials and Methods}

\section{Specimen Preparation}

This study was performed after obtaining approval from the Institutional Review Board. Ten paired fresh-frozen cadaveric knees (total 20 knees) from 10 donors (seven males and three females; mean age at the time of death, $62.4 \pm 4.9$ years) were used (Table 1). All knees were grossly intact and had no evidence of advanced degenerative changes, structural deformities, ligament instability, or history of previous surgery. The specimens were kept frozen

Table 1. Demographic Characteristics of Cadavers

\begin{tabular}{llcccc}
\hline ID & \multicolumn{1}{c}{ Sex } & $\begin{array}{c}\text { Age } \\
(\mathrm{yr})\end{array}$ & $\begin{array}{c}\text { Height } \\
(\mathrm{m})\end{array}$ & $\begin{array}{c}\text { Weight } \\
(\mathrm{kg})\end{array}$ & $\begin{array}{c}\mathrm{BMI} \\
\left(\mathrm{kg} / \mathrm{m}^{2}\right)\end{array}$ \\
\hline$\# 1$ & Male & 66 & 1.69 & 74 & 25.9 \\
$\# 2$ & Male & 62 & 1.71 & 60 & 20.5 \\
$\# 3$ & Female & 61 & 1.54 & 55 & 23.2 \\
$\# 4$ & Male & 64 & 1.74 & 79 & 26.1 \\
$\# 5$ & Female & 54 & 1.52 & 50 & 21.6 \\
$\# 6$ & Male & 66 & 1.76 & 70 & 22.6 \\
$\# 7$ & Male & 64 & 1.78 & 80 & 25.2 \\
$\# 8$ & Male & 60 & 1.75 & 74 & 24.2 \\
$\# 9$ & Male & 71 & 1.73 & 76 & 25.4 \\
$\# 10$ & Female & 56 & 1.55 & 56 & 23.3 \\
Overall & Male (7), & $62.4 \pm 4.9$ & $1.67 \pm 0.10$ & $67.4 \pm 11.1$ & $23.8 \pm 1.9$ \\
& female (3) & & & & \\
\hline
\end{tabular}

Values are presented as mean \pm standard deviation. BMI: body mass index. at $-20^{\circ} \mathrm{C}$ before the experiment and were thawed for 1 day at room temperature. The specimens were cut to $20 \mathrm{~cm}$ in length from the joint line. All soft tissues, except the ACL and PCL, were removed carefully from the femur and tibia. Each bundle of the ACL (anteromedial $[\mathrm{AM}]$ and posterolateral [PL] bundles) and PCL (anterolateral $[\mathrm{AL}]$ and posteromedial [PM] bundles) was identified and subsequently separated observing differences in the orientation of the bundle fibers and tensioning with passive knee range of motion. The knee joints were disarticulated after transecting the ACL and PCL at their mid-substance. The outer borders of the femoral and tibial insertion sites of the ACL and PCL were marked with a dark ink marker after removing the fibers from the bony footprints. The marked ink borders were later covered with white paint to make the borders more prominent when digitizing them with a 3D digitizer. Three registration spheres (15 mm diameter; BNB, Seoul, Korea) were attached to the proximal tibia and another three to the distal femur for later registration of bony surface and the ACL and PCL insertion sites. The spheres were placed leaving an interval of approximately 3 $\mathrm{cm}$ distal to tibial tuberosity on the tibia and proximal to adductor tubercle on the femur.

\section{Digitization of the Outer Borders of the ACL and PCL/3D Surface Scanning of the Femur and Tibia}

The 3D spatial positions of the outer border of each ACL and PCL bundle and the three registration spheres on the femur and tibia were digitized using a 3D digitizer (MiscroScribe-G2; Immersion, San Jose, CA, USA) and a computer-assisted design remodeling program (Rhinoceros 4.0; Robert McNeal \& Associates, Seattle, WA, USA). More than 20 different points on the spheres were digitized to validate the accuracy of digitization. The measured diameter of the sphere was $15.1 \pm 0.1 \mathrm{~mm}$. The difference between the known and measured diameter of the sphere was $0.1 \pm 0.1 \mathrm{~mm}$. The geometric centroid of the points was calculated using the $\mathrm{x}, \mathrm{y}$, and $\mathrm{z}$ coordinates and centroid formula in the Rhinoceros program for later matching with the center coordinate of the scanned registration sphere. Then the positional data were saved separately for each specimen.

After digitization, all bony specimens (femur and tibia) underwent outer surface scanning using a 3D scanner (Rexcan 3; Solutionix, Seoul, Korea) while maintaining all of the registration spheres (Fig. 1). This 3D scanner used twin-cameras and a whitelight phase-shifting triangulation technique to achieve high resolution and accuracy $(<10 \mu \mathrm{m})$ with decreased data noise. Each specimen was fixed on a special turntable approximately $1 \mathrm{~m}$ in front of the scanner. We could obtain reliable surface scanning 

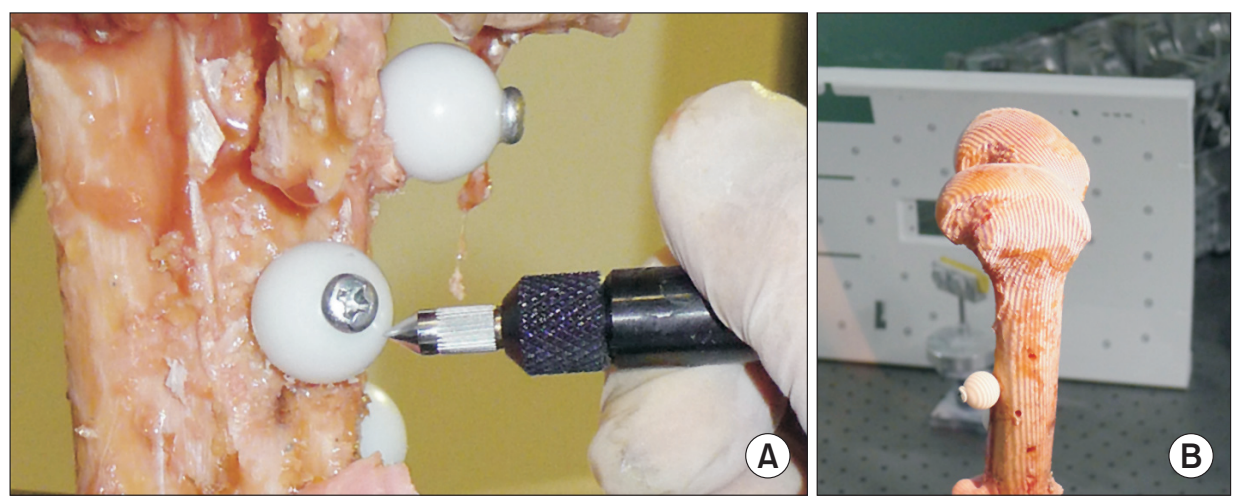

Fig. 1. Digitization of the registration spheres using a three-dimensional (3D) digitizer (A) and outer surface scanning of the distal femur using a 3D scanner (B).

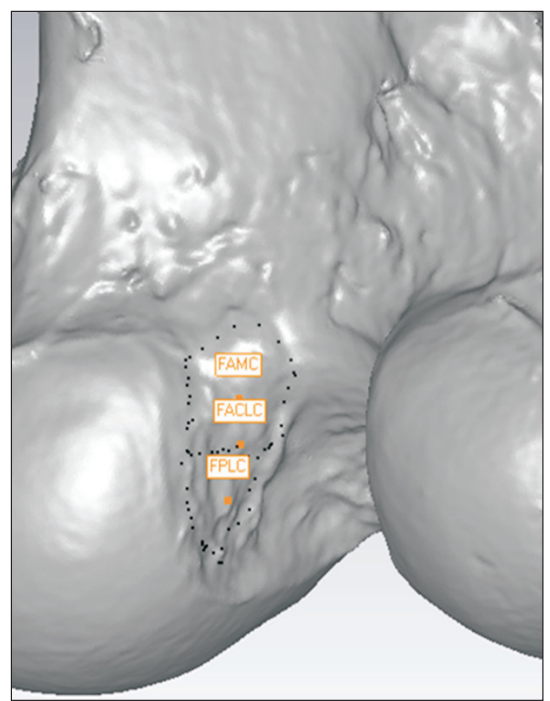

Fig. 2. Superimposition of the data and calculation of the footprint centroids of the ACL (anteromedial, posterolateral, and entire bundles) in specialized computer software. The dotted line is a footprint margin of the ACL. ACL: anterior cruciate ligament, FAMC: femoral anteromedial bundle centroid, FPLC: femoral posterolateral bundle centroid, FACLC: femoral anterior cruciate ligament entire bundle centroid.

data because the turntable, which rotates $360^{\circ}$ around one axis, caused no vibration during scanning.

\section{Superimposition of the Data/Calculation of the Footprint Centers of the ACL and PCL}

Geomagic Studio v10.0 (Research Triangle Park, NC, USA) was used to superimpose the acquired data. The scanned bony surface data and the sphere centroids with the digitized insertion site data were opened in the software. The geometric centroid coordinates of the spheres in the scanned data were calibrated using the coordinates and centroid formula for 3D object. By registering each geometric centroid of the spheres in the scanned data to the corresponding geometric centroid of the spheres in the digitized data of the surface model, the outer borders of the insertion sites

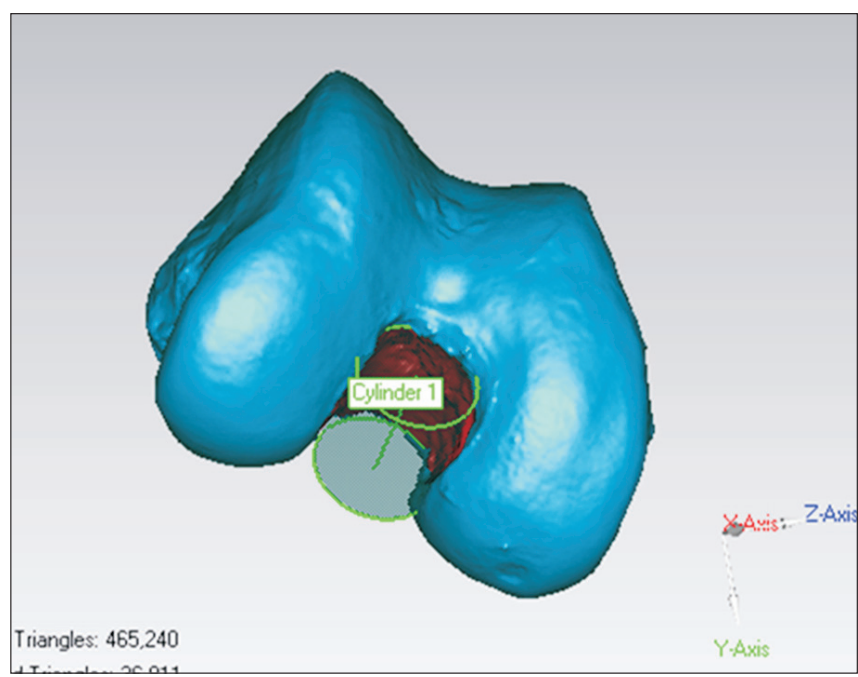

Fig. 3. Measurement of the notch diameter (intercondylar notch width). We created a cylinder that fits the intercondylar notch using a tool in Geomagic software and presented the width of the intercondylar notch by the diameter of the cylinder.

were displayed on the scanned bony surface data. Using the tool to calculate the geometric centroid in the Geomagic program, the center coordinates of the ACL (AM, PL, and entire ACL bundle) footprints and PCL (AL, PM, and entire PCL bundle) footprints were calculated and marked on the scanned bony surfaces (Fig. 2).

\section{Measurement of Side-to-Side Differences}

We evaluated various 3D morphometric parameters using the reprocessed data in Geomagic software. To compare the right and left distal femurs, we measured anterior-posterior (AP) length of the medial femoral condyle, AP length of the lateral femoral condyle, transepicondylar length, width of the intercondylar notch, footprint areas of the ACL and PCL, and distal femoral volume. Each length was measured at the widest part on the scanned data considering 3D morphology and each coordinate. The distal femur can be divided into medial and lateral condyles with the in- 
tervening intercondylar notch, and we measured the parameters on both sides. Then, we measured the footprint areas of the ACL and PCL, which were the most prominent soft tissue structures in the knee joint. We created a cylinder that fits the width of the intercondylar notch using the Geomagic program tool (create 3D feature), which creates a cylinder feature on a point or polygon object, based on an existing polygon structure or parametric input. We presented the width as a diameter of the cylinder (Fig. 3). The footprint areas of the ACL and PCL on the distal femur and proximal tibia were measured using a measuring tool (select by area) in Geomagic software. This tool measured the surface area inside of the defined footprint border taking the rise and fall of the surface into consideration. We assessed volume from the distal end of the femur to the adductor tubercle ${ }^{16)}$. The Euclidean distances between any two points on the models were measured

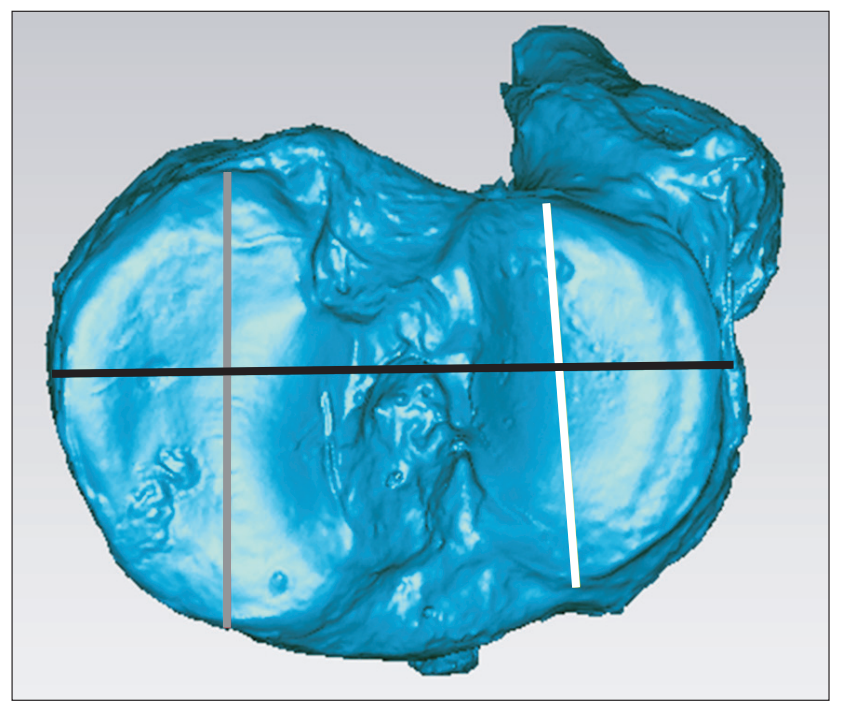

Fig. 4. Measurement of various parameters in the proximal tibia in Geomagic software. White line: anteroposterior length of the lateral tibial plateau, gray line: anteroposterior length of the medial tibial plateau, black line: total width of the tibial plateau. using a measuring tool (datum distance measure) by calculating the distance between two points by the Pythagorean formula relative to the coordinate system in Geomagic software.

AP length of the medial and lateral tibial plateaus, total width (medial to lateral width) of the tibial plateau, ACL and PCL footprint areas, and proximal tibial volume were measured to compare the right and left proximal tibiae (Fig. 4). As the medial and lateral condyles of the tibia also have different morphologies, AP lengths of the medial and lateral tibial plateau were measured respectively. We assessed volume from proximal end of the tibia to distal end of the tibial tuberosity for consistency when measuring proximal tibial volume (Fig. 5).

We compared the 3D bony shape and the location of the center of the cruciate ligament footprint on opposite sides by making a mirror model of the left-side surface scanned model. This mirror model was superimposed on the right-side scanned model. We calculated the average deviation of $3 \mathrm{D}$ alignment between

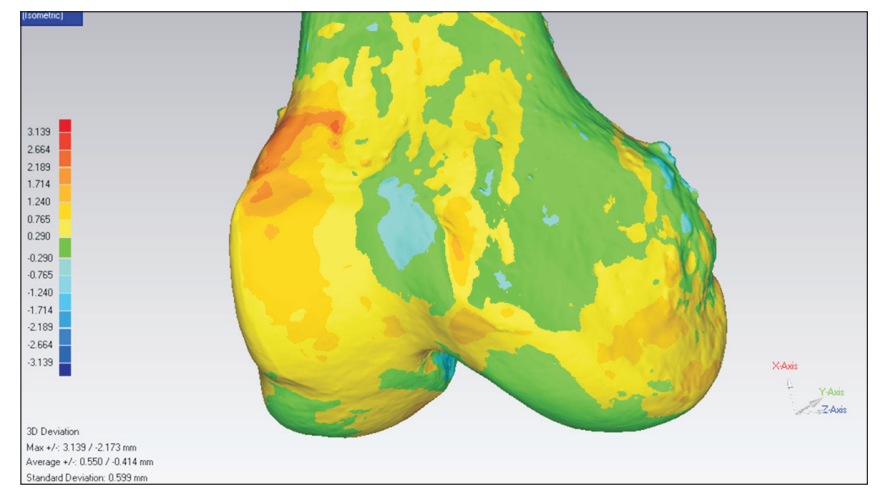

Fig. 6. Three-dimensional side-to-side morphological differences. A mirror model of the surface scanned model of the left side was made. This mirror model was superimposed on the right side scanned model. Using a special tool in Geomagic software, the average deviation of alignment between the right side model and left side model was calculated. In addition, bony shape differences were displayed as a continuous color map.

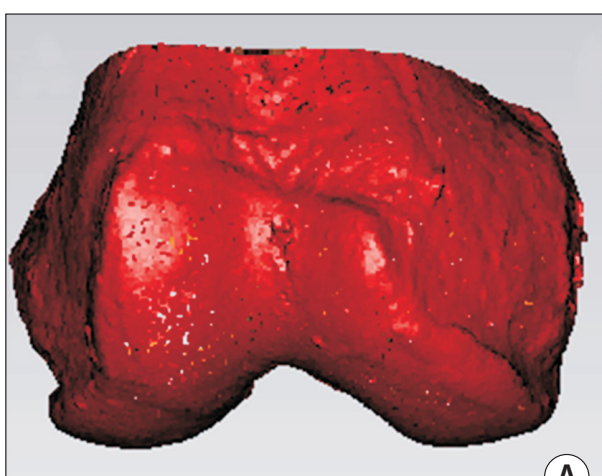

(A)

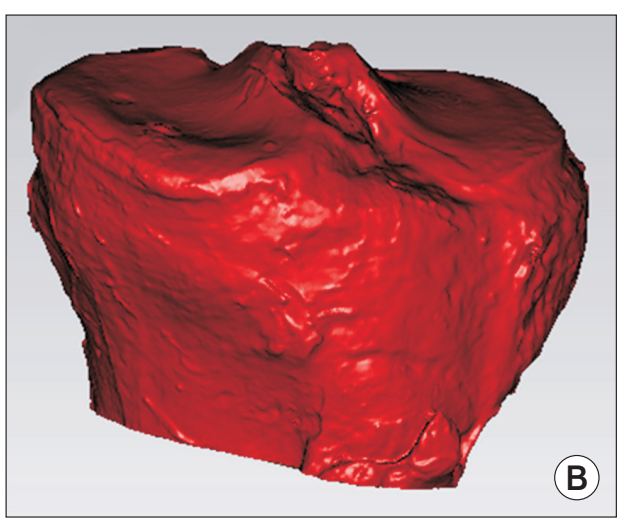

(B)
Fig. 5. Three-dimensional volume measurement of the distal femur (A) and proximal tibia (B) using a tool in Geomagic software. The distal femoral volume was assessed as a volume from the distal end of the femur to the adductor tubercle. The proximal tibial volume was assessed as a volume from the proximal end of the tibia to the distal end of the tibial tuberosity. 
the right-side model and a mirror model of the left-side model using the best fit alignment tool in the Geomagic software (Fig. 6). First, we selected the right-side model as a fixed object and the left-side model as a floating object. Then, the two models were brought into a roughly aligned (matched) position. The test model was sampled and the closest points were computed on the reference to each sample point based on the sample size selected in the command. The sums of squares of distances between the sample pairs were minimized through the least-squares method, which is a mathematical procedure for finding the best-fitting line or curve to a given set of points by minimizing the sum of the squares of the residuals (offsets) of the points from the line or curve. The sum of the squares of the offsets is used instead of the offset absolute values because this allows the residuals to be treated as a continuous differentiable quantity ${ }^{17)}$. The number of points on the surface of each model was specified as 1,500 for precise alignment. Last, deviations at all points were compared, and the average of the deviations was calculated as the average deviation of alignment between the right- and left-side models. After overlapping the two models, we measured the distances between the center coordinates of the right ACL (AM, PL, and entire ACL bundle) and PCL (AL, PM, and entire PCL bundle) footprints and those of the left ACL and PCL footprints. All measurements including all Euclidean distances, areas, and volumes in 20 knees were conducted with a precision of $0.1 \mathrm{~mm}$ by one observer and were recorded twice with an interval of 2 weeks.
The mean values were used for data analysis.

\section{Statistical Analysis}

Based on parameters used in a previous side-to-side difference comparison study ${ }^{1)}$, the minimum sample size for both groups was calculated as 10 for a power of 0.8 to detect $50 \%$ difference at the 0.05 level. The post-hoc power calculated by given $\alpha$-error, total sample size, and effect size was 0.8. A normal data distribution was validated using the Kolmogorov-Smirnov procedure. A paired $t$-test was used to evaluate overall differences in morphometric parameters, including all measured lengths, areas, volumes, and alignment deviations between right and left knees. A p-value $<0.05$ was considered significant. To assess the testretest reproducibility, intraclass correlation coefficient (ICC) was calculated with $95 \%$ confidence intervals for all measurements including Euclidean distances between any two points, areas, and volumes. Data analysis was performed with SPSS ver. 15.0 (SPSS Inc., Chicago, IL, USA).

The absolute percentage of side-to-side differences was calculated to verify whether individual side-to-side differences were smaller than the inter-subject variability. The mean percentage of side-to-side difference was compared to the coefficient of variation (standard deviation divided by the mean) of the 10 specimens. The mean percentage of side-to-side difference indicates the intra-subject variability, and the coefficient of variation indicates the inter-subject variation. According to Eckstein et al.,

Table 2. Parameters Measured from the Distal Femur

\begin{tabular}{lcccccc}
\hline \multicolumn{1}{c}{ Parameter } & Right & Left & ICC (95\% CI) & $\begin{array}{c}\text { Mean percentage } \\
\text { of side-to-side } \\
\text { difference }\end{array}$ & $\begin{array}{c}\text { Coefficient } \\
\text { of variation }\end{array}$ & $\begin{array}{c}\text { Ratio of } \\
\text { variation }\end{array}$ \\
& & & & & & \\
p-value
\end{tabular}

Values are presented as mean \pm standard deviation.

ICC: intraclass correlation coefficient, CI: confidence interval, ACL: anterior cruciate ligament, AM: anteromedial, PL: posterolateral, PCL: posterior cruciate ligament, AL: anterolateral, PM: posteromedial.

a) $\mathrm{p}$-value $<0.05$ was considered significant. 
the ratio between the mean percentage of side-to-side difference and the coefficient of variation (ratio of variation) $>1$ indicated that inter-subject variability was larger than the individual sideto-side difference ${ }^{2)}$.

\section{Results}

The ICCs for test-retest reliability were greater than 0.9 for all measurements. No significant differences were observed between any measurements taken from the distal femur of the right and left knees. For all length parameters of the distal femur, side-toside differences were $<1 \mathrm{~mm}$. Total areas of the ACL footprints were $171.4 \pm 12.6 \mathrm{~mm}^{2}$ on the right side and $167.5 \pm 9.2 \mathrm{~mm}^{2}$ on the left side ( $\mathrm{p}=0.527)$. Total areas of the PCL footprints were $271.4 \pm 15.9 \mathrm{~mm}^{2}$ on the right side and $269.5 \pm 19.5 \mathrm{~mm}^{2}$ on the left side $(\mathrm{p}=0.845)$. Total volumes of the distal femurs were $148.5 \pm 9.0$ $\mathrm{cm}^{3}$ on the right side and $146.2 \pm 10.4 \mathrm{~cm}^{3}$ on the left side $(\mathrm{p}=0.660)$ (Table 2). The maximal side-to-side difference in distal femoral volume was approximately $6.5 \mathrm{~cm}^{3}$.

No significant differences were observed between any measurements taken from the proximal tibia of the right and left knees. For all length parameters, side-to-side differences were $<1 \mathrm{~mm}$. Total areas of the ACL footprints were $202.9 \pm 10.4 \mathrm{~mm}^{2}$ on the right side and $199.1 \pm 8.6 \mathrm{~mm}^{2}$ on the left side $(\mathrm{p}=0.500)$. Total areas of the PCL footprints were $177.8 \pm 12.2 \mathrm{~mm}^{2}$ on the right side and $174.5 \pm 9.9 \mathrm{~mm}^{2}$ on the left side $(\mathrm{p}=0.617)$. Total volumes of the proximal tibiae were $130.5 \pm 11.2 \mathrm{~cm}^{3}$ on the right side and
$130.9 \pm 12.1 \mathrm{~cm}^{3}$ on the left side ( $\mathrm{p}=0.953$ ) (Table 3). The maximal side-to-side volume difference in the proximal tibia was approximately $9.6 \mathrm{~cm}^{3}$.

Average deviation of 3D alignment between the right and left sides was $0.8 \pm 0.4 \mathrm{~mm}$ for the distal femur and $1.1 \pm 0.6 \mathrm{~mm}$ for the proximal tibia. The center-to-center distances between the right and left ligament footprints were approximately $2 \mathrm{~mm}$ for both ACL and PCL footprints. No significant differences were observed in the center-to-center distances between the tibia and femur (Table 4).

Table 4. Center-to-Center Distance of Each Bundle between the Right and Left Knees during Overlapping

\begin{tabular}{lccc}
\hline \multicolumn{1}{c}{ Variable } & Femur & Tibia & p-value \\
\hline ACL & & & \\
AM center to AM center (mm) & $2.6 \pm 0.4$ & $2.7 \pm 0.5$ & 0.574 \\
PL center to PL center (mm) & $2.4 \pm 0.7$ & $2.8 \pm 0.8$ & 0.370 \\
ACL center to ACL center (mm) & $2.5 \pm 0.5$ & $2.8 \pm 0.6$ & 0.475 \\
PCL & & & \\
AL center to AL center $(\mathrm{mm})$ & $1.9 \pm 1.2$ & $1.5 \pm 0.6$ & 0.490 \\
PM center to PM center $(\mathrm{mm})$ & $2.2 \pm 1.4$ & $1.8 \pm 0.9$ & 0.596 \\
PCL center to PCL center $(\mathrm{mm})$ & $2.3 \pm 1.1$ & $1.8 \pm 0.5$ & 0.349 \\
\hline
\end{tabular}

Values are presented as mean \pm standard deviation.

ACL: anterior cruciate ligament, AM: anteromedial, PL: posterolateral, PCL: posterior cruciate ligament, AL: anterolateral, PM: posteromedial. ${ }^{a}$ p-value $<0.05$ was considered significant.

Table 3. Parameters Measured from the Proximal Tibia

\begin{tabular}{lcccccc}
\hline \multicolumn{1}{c}{ Parameter } & Right & Left & ICC (95\% CI) & $\begin{array}{c}\text { Mean percentage } \\
\text { of side-to-side } \\
\text { difference }\end{array}$ & $\begin{array}{c}\text { Coefficient } \\
\text { of variation }\end{array}$ & $\begin{array}{c}\text { Ratio of } \\
\text { variation }\end{array}$ \\
& & & & & & \\
p-value
\end{tabular}

Values are presented as mean \pm standard deviation.

ICC: intraclass correlation coefficient, CI: confidence interval, ACL: anterior cruciate ligament, AM: anteromedial, PL: posterolateral, PCL: posterior cruciate ligament, AL: anterolateral, PM: posteromedial.

${ }^{\text {a) }}$-value $<0.05$ was considered significant. 
Ratios of variation in all tibial and femoral measurements were $>1$, indicating that inter-subject variability was larger than the individual side-to-side differences (Tables 2 and 3).

\section{Discussion}

The most important findings of this study were the lack of significant differences in the 3D morphometric measurements between right and left knees, and the fact that the inter-subject variability was remarkably larger than the within-subject side-toside differences. We conducted cadaveric measurements using a $3 \mathrm{D}$ digitizer, surface scanning, and specialized software to generate more accurate results than previous 2D methods. In addition, fresh-frozen cadaveric knees were used and surface scanning was readily performed to minimize tissue deformation.

Only a few reports have been published on side-to-side differences in knee joints. Dargel et al. ${ }^{1)}$ reported that only three dimensions (anatomical lateral distal femoral angle, tibial posterior slope, and intercondylar roof inclination) differed significantly among 71 morphometric dimensions measured about the knee. They concluded that there was a good correlation in the morphometric dimensions of the human knee joint between the right and left sides. They measured detailed parameters including the femur, tibia, cruciate ligaments, and menisci using 20 pairs of formalin-fixed human cadaveric knees. Although they measured a variety of parameters, they used 2D methods with calipers and simple radiography. Eckstein et al. ${ }^{2)}$ investigated differences in the articular surface area and volume, as well as local cartilage thickness between the right and left knees using magnetic resonance imaging (MRI). Those authors supported using reference data from the non-involved side to estimate cartilage loss in patients with unilateral osteoarthritis. Teitz et al. ${ }^{18)}$ reported no significant difference between right and left knees in the notch width index. However, these previous studies were based on 2D morphometric analysis with some limitations to interpret the exact $3 \mathrm{D}$ form.

The previous methods provided a $2 \mathrm{D}$ representation of a $3 \mathrm{D}$ object; thus, they remain inaccurate even after properly correcting for magnification, measurement technique, and projection ${ }^{19)}$. In the present study, all morphometric dimensions were measured three-dimensionally. The volumes of the distal femur and proximal tibia and the total ACL and PCL footprint areas were measured using special tools in Geomagic software, and all length measurements were calculated by measuring the $3 \mathrm{D}$ distances between two points. In addition, 3D deviations were measured using a special tool in the software.

Some investigators have already conducted actual measure- ments or 3D analysis for the morphometric side-to-side comparison of the knee joints. Terzidis et al..$^{9)}$ conducted actual measurements in a large sample of dried femori using a digital sliding caliper. They measured the bicondylar width, medial and lateral femoral condylar width, intercondylar width, and intercondylar depth on dried femoral bones and examined side-to-side difference. Although they conducted actual measurements, they measured and compared only the distances without conducting 3D analysis. Jamison et al. ${ }^{11)}$ measured the volume of the ACL using an MRI-based method and conducted side-to-side comparison. The volume of the ACL was measured using MRI segmentation technique, which had been previously validated using porcine ACLs with a 0.98 correlation between MRI-based ACL volume and true $\mathrm{ACL}$ volume $\mathrm{e}^{20}$. However, this technique requires manual segmentation of the ACL from the MRI. It could be sometimes very difficult to distinguish the ACL from surrounding tissues. In other words, the results of segmentation technique depend on the quality of the MRI and experiences of the examiner. Different from previous methods, we 3-dimensionally measured the parameters including distance, area, and volume in fresh-frozen cadaveric knees using specialized software with high accuracy. A recent study demonstrated that segmentation of computed tomography (CT) and MRI images of long bones showed a root mean square (RMS) error of $0.55 \mathrm{~mm}$ and $0.56 \mathrm{~mm}$, respectively ${ }^{21)}$. In contrast, mean RMS errors of the Geomagic software were mostly $<10^{-5} \mu \mathrm{m}$ in a test by National Institute of Standards and Technology (NIST Test No. 681/280055-10). Thus, measurements in this study might be more accurate to evaluate morphometric side-to-side differences. However, further studies that compare our method with CT or MRI might be necessary to elucidate the measurement accuracy and precision.

The present study showed remarkable 3D symmetry between left and right knee joints. The side-to-side differences in length dimensions were less than $1 \mathrm{~mm}$, and the average deviations of $3 \mathrm{D}$ alignment of the proximal tibia and distal femur were only approximately $1 \mathrm{~mm}$. Considering the precision of our method, the magnitude of these differences might be a reliable representation of similarity between the right and left knee joints. In addition, our analysis of symmetry in healthy knees may suggest a new and potentially more accurate method to compare symmetry in knees with osteoarthritis.

Total footprint areas did not differ between right and left knees as well as the osseous morphology. However, the distances between the footprint centers of the right and left sides were approximately $2 \mathrm{~mm}$ or a little larger for all ACL and PCL bundles. Differences in cruciate ligament footprints between right and 
left knees seemed to be large compared to similarities in osseous morphology. However, these 3D results showed smaller sideto-side differences compared to previous $2 \mathrm{D}$ reports, which presented an approximately $4 \mathrm{~mm}$ difference in distance ${ }^{8)}$. The importance of anatomical reconstruction has been recently emphasized for cruciate ligament reconstruction ${ }^{22}$. Surgeons may be able to achieve more functional restoration of the ACL and PCL using anatomical reconstruction ${ }^{12,22-24)}$. A recent study showed that incorrect bone tunnel position was the main cause for persistent instability and graft failure ${ }^{23)}$. If side-to-side differences at the cruciate ligament footprints are minimal, improved bone tunnel position could be achieved by referring to the uninjured side anatomy. Using 3D measurements, we found 2-3 mm differences in the location of the center of right and left ACL and PCL bundle footprints. Thus, we think that the surgeon should strive toward close anatomical approximation using contralateral cruciate ligament geometry, particularly during computer-assisted surgical procedures.

The present study has some limitations. First, the number of human cadaveric knees was too small for generalization, and specimens' ages were relatively old. However, we did not find any moderate or advanced arthritic changes in the specimens. Second, tissue shrinkage can occur in cadaveric specimens during the time for digitization and surface scanning. Thus, we tried to reduce this duration, and scanning of the specimens was performed promptly. Furthermore, as both sides were tested in the same condition, tissue shrinkage may not have affected the final results. Third, some specimens had indefinite margins for outer borders of the ACL and PCL, making correct delineation of attachment sites of each ACL and PCL bundle difficult to achieve in some cases. Thus, we passed a suture into the remnant cruciate bundle approximately $1 \mathrm{~cm}$ from the footprints. The sutures were tensioned to identify the fibers and margins more definitely during marking of the footprint margins. With this method, we might have been able to reduce the errors as in a previous anatomic study ${ }^{25)}$. All authors participated in the observational assessment and identification of the outer margins, and the margins were decided under agreement of all authors. Fourth, we could not determine the RMS errors of the Microscribe and Geomagic studio system during digitization, transformation, and 3D scanning. However, in a study of the accuracy test for the Microscribe system, the authors were confident that the RMS error is within $1 \mathrm{~mm}^{26)}$. Regarding the Geomagic software, we could find a result by National Institute of Standards and Technology (NIST Test No. 681/280055-10). The least-squares fitting features of the Geomagic software were tested on 240 data sets, representing the following geometry types: lines, planes, spheres, cylinders and cones. Mean RMS errors for these geometric types were mostly $<10^{-5} \mu \mathrm{m}$ except cones with 0.4 to $7 \mu \mathrm{m}$, and the expanded uncertainty for each geometric type was less than $1.0 \times 10^{-9}$. Although we could not calculate exact RMS errors and cumulative effects of them, we think that our method using the Microscribe and Geomagic studio might be accurate enough to assess the side-toside differences of the knee joints. However, considering more complex geometries in our investigation than those in the NIST test, there could be some differences in RMS errors. Fifth, the methods used in the current study could not be reproduced clinically. Thus, an intermediate study might be necessary to assess differences in side-to-side mapping using non-invasive methods such as MRI or CT. Lastly, we conducted 3D evaluation only for the bony structures of right and left knee joints. Future studies that compare muscle mass, muscle strength, and potential laxity would be necessary to assess side-to-side differences in functional properties of the right and left knees.

\section{Conclusions}

In conclusion, we conducted 3D measurements of the human cadaveric knee joints, and our results revealed that 3D morphology in healthy knee joints is remarkably symmetrical between right and left knees. Our results might support the concept of obtaining morphologic reference data from the uninvolved contralateral knee, particularly for bony surgeries including arthroplasties. However, considering the injured or osteoarthritic knees commonly have deformities, further studies are required to elucidate whether the contralateral knee joints can be used for surgical planning or mapping.

\section{Conflict of Interest}

No potential conflict of interest relevant to this article was reported.

\section{References}

1. Dargel J, Feiser J, Gotter M, Pennig D, Koebke J. Side differences in the anatomy of human knee joints. Knee Surg Sports Traumatol Arthrosc. 2009;17:1368-76.

2. Eckstein F, Muller S, Faber SC, Englmeier KH, Reiser M, Putz R. Side differences of knee joint cartilage volume, thickness, and surface area, and correlation with lower limb dominance: an MRI-based study. Osteoarthritis Cartilage. 
2002;10:914-21.

3. Gokeler A, Schmalz T, Knopf E, Freiwald J, Blumentritt S. The relationship between isokinetic quadriceps strength and laxity on gait analysis parameters in anterior cruciate ligament reconstructed knees. Knee Surg Sports Traumatol Arthrosc. 2003;11:372-8.

4. Jordan SS, DeFrate LE, Nha KW, Papannagari R, Gill TJ, Li G. The in vivo kinematics of the anteromedial and posterolateral bundles of the anterior cruciate ligament during weightbearing knee flexion. Am J Sports Med. 2007;35:547-54.

5. Li G, Papannagari R, Li M, Bingham J, Nha KW, Allred D, Gill T. Effect of posterior cruciate ligament deficiency on in vivo translation and rotation of the knee during weightbearing flexion. Am J Sports Med. 2008;36:474-9.

6. Papannagari R, Gill TJ, Defrate LE, Moses JM, Petruska AJ, Li G. In vivo kinematics of the knee after anterior cruciate ligament reconstruction: a clinical and functional evaluation. Am J Sports Med. 2006;34:2006-12.

7. Shefelbine SJ, Ma CB, Lee KY, Schrumpf MA, Patel P, Safran MR, Slavinsky JP, Majumdar S. MRI analysis of in vivo meniscal and tibiofemoral kinematics in ACL-deficient and normal knees. J Orthop Res. 2006;24:1208-17.

8. Dargel J, Pohl P, Tzikaras P, Koebke J. Morphometric side-toside differences in human cruciate ligament insertions. Surg Radiol Anat. 2006;28:398-402.

9. Terzidis I, Totlis T, Papathanasiou E, Sideridis A, Vlasis K, Natsis K. Gender and side-to-side differences of femoral condyles morphology: osteometric data from 360 caucasian dried femori. Anat Res Int. 2012;2012:679658.

10. Scanlan SF, Lai J, Donahue JP, Andriacchi TP. Variations in the three-dimensional location and orientation of the ACL in healthy subjects relative to patients after transtibial ACL reconstruction. J Orthop Res. 2012;30:910-8.

11. Jamison ST, Flanigan DC, Nagaraja HN, Chaudhari AM. Side-to-side differences in anterior cruciate ligament volume in healthy control subjects. J Biomech. 2010;43:576-8.

12. Zhu W, Lu W, Han Y, Hui S, Ou Y, Peng L, Fen W, Wang D, Zhang L, Zeng Y. Application of a computerised navigation technique to assist arthroscopic anterior cruciate ligament reconstruction. Int Orthop. 2013;37:233-8.

13. Kraus MD, Dehner C, Riepl C, Scholl H, Gebhard F. A novel method of image-based navigation in fracture surgery. Arch Orthop Trauma Surg. 2012;132:741-50.

14. Hernandez-Vaquero D, Suarez-Vazquez A, Iglesias-Fernandez S. Can computer assistance improve the clinical and functional scores in total knee arthroplasty? Clin Orthop
Relat Res. 2011;469:3436-42.

15. Hitt K, Shurman JR 2nd, Greene K, McCarthy J, Moskal J, Hoeman T, Mont MA. Anthropometric measurements of the human knee: correlation to the sizing of current knee arthroplasty systems. J Bone Joint Surg Am. 2003;85 Suppl 4: 115-22.

16. Iacono F, Lo Presti M, Bruni D, Raspugli GF, Bignozzi S, Sharma B, Marcacci M. The adductor tubercle: a reliable landmark for analysing the level of the femorotibial joint line. Knee Surg Sports Traumatol Arthrosc. 2013;21:2725-9.

17. Markovsky I, Van Huffel S. Overview of total least-squares methods. Signal Process. 2007;87:2283-302.

18. Teitz CC, Lind BK, Sacks BM. Symmetry of the femoral notch width index. Am J Sports Med. 1997;25:687-90.

19. Anderson AF, Anderson CN, Gorman TM, Cross MB, Spindler KP. Radiographic measurements of the intercondylar notch: are they accurate? Arthroscopy. 2007;23:261-8.

20. Chaudhari AM, Zelman EA, Flanigan DC, Kaeding CC, Nagaraja HN. Anterior cruciate ligament-injured subjects have smaller anterior cruciate ligaments than matched controls: a magnetic resonance imaging study. Am J Sports Med. 2009;37:1282-7.

21. Van den Broeck J, Vereecke E, Wirix-Speetjens R, Vander Sloten J. Segmentation accuracy of long bones. Med Eng Phys. 2014;36:949-53.

22. Yasuda K, van Eck CF, Hoshino Y, Fu FH, Tashman S. Anatomic single- and double-bundle anterior cruciate ligament reconstruction, part 1: basic science. Am J Sports Med. 2011; 39:1789-99.

23. Meredick RB, Vance KJ, Appleby D, Lubowitz JH. Outcome of single-bundle versus double-bundle reconstruction of the anterior cruciate ligament: a meta-analysis. Am J Sports Med. 2008;36:1414-21.

24. Zantop T, Petersen W, Sekiya JK, Musahl V, Fu FH. Anterior cruciate ligament anatomy and function relating to anatomical reconstruction. Knee Surg Sports Traumatol Arthrosc. 2006;14:982-92.

25. Ziegler CG, Pietrini SD, Westerhaus BD, Anderson CJ, Wijdicks CA, Johansen S, Engebretsen L, LaPrade RF. Arthroscopically pertinent landmarks for tunnel positioning in single-bundle and double-bundle anterior cruciate ligament reconstructions. Am J Sports Med. 2011;39:743-52.

26. Pandya A, Siadat MR, Auner G. Design, implementation and accuracy of a prototype for medical augmented reality. Comput Aided Surg. 2005;10:23-35. 